\title{
Effect of lead acetate on neurobehavioral development of rats
}

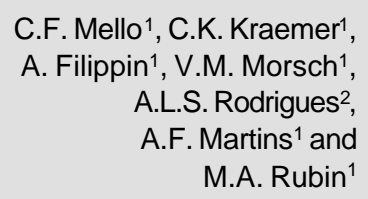

\author{
1Departamento de Química, Centro de Ciências Naturais e Exatas, \\ Universidade Federal de Santa Maria, Santa Maria, RS, Brasil \\ Departamento de Bioquímica, Centro de Ciências Biológicas, \\ Universidade Federal de Santa Catarina, Florianópolis, SC, Brasil
}

Correspondence
C.F. Mello
Departamento de Química
CCNE, UFSM
$97105-900$ Santa Maria, RS
Brasil
Fax: 55 (055) 220-8031
E-mail: cfmello@base.ufsm.br
Research supported by CNPq,
CAPES,FAPERGS,FIPE-UFSM.
C.F. Mello (No. 351127/97-6) and
M.A. Rubin (No. 520189/97-3) were
recipients of CNPq fellowships,
and C.K. Kraemer and A. Filippin
of FIPE-UFSM fellowships.
. . . . . . . . . . . . . . . . . . . . .

Received September 11, 1997 Accepted April 14, 1998

\section{Abstract}

We investigated the effects of lead exposure during the pre- and postnatal period on the neurobehavioral development of female Wistar rats (70-75 days of age, 120-150 g) using a protocol of lead intoxication that does not affect weight gain. Wistar rats were submitted to lead acetate intoxication by giving their dams $1.0 \mathrm{mM}$ lead acetate. Control dams received deionized water. Growth and neuromotor development were assessed by monitoring daily the following parameters in 20 litters: body weight, ear unfolding, incisor eruption, eye opening, righting, palmar grasp, negative geotaxis, cliff avoidance and startle reflex. Spontaneous alternation was assessed on postnatal day 17 using a T maze. The animals' ability to equilibrate on a beaker rim was measured on postnatal day 19. Lead intoxication was confirmed by measuring renal, hepatic and cerebral lead concentration in dams and litters. Lead treatment hastened the day of appearance of the following parameters: eye opening (control: $13.5 \pm 0.6, \mathrm{~N}=88$; lead: $12.9 \pm 0.6$, $\mathrm{N}=72 ; \mathrm{P}<0.05$ ), startle reflex (control: $13.0 \pm 0.8, \mathrm{~N}=88$; lead: 12.0 $\pm 0.7, \mathrm{~N}=72 ; \mathrm{P}<0.05$ ) and negative geotaxis. On the other hand, spontaneous alternation performance was hindered in lead-exposed animals (control: $37.6 \pm 19.7$; lead: $57.5 \pm 28.3 \%$ of alternating animals; $\mathrm{P}<0.05$ ). These results suggest that lead exposure without concomitant undernutrition alters rat development, affecting specific subsets of motor skills.

\section{Introduction}

Lead is among the more common toxic metals present in our environment. The primary site of action of lead is the central nervous system, and exposure to the metal is associated with several neurobehavioral and psychological alterations (1-4). Neurochemical alterations induced by lead include agespecific changes in the level of binding of
Key words

- Lead

- Neurobehavioral

development

- Undernutrition

- Heavy metal intoxication

- Teratology

- Rat glutamatergic and cholinergic ligands (5-7), competition with $\mathrm{Ca}^{2+}$ and direct stimulation of calmodulin (8), reduced effects of depolarization on $\mathrm{Ca}_{\mathrm{i}}{ }^{2+}$ levels (9), and increased liperoxidative damage in brain (10).

The developmental toxicity of lead has become a significant area of research since children are much more sensitive than adults to learning impairment following low-level lead exposure (11). Regarding this point, animal 
and human studies have reached similar results, since behavioral effects induced by lead are seen in rats at approximately the same blood levels that cause deficits in humans $(3,12,13)$.

Administration of diets with a high lead content to rodents during suckling causes growth impairment, hematological alterations, breakdown of the blood-brain barrier, and higher pup mortality $(14,15)$. Moreover, significant effects on neuromotor development of rats have also been described. Reiter and colleagues (16) reported delay in development of righting reflex and eye opening in animals exposed to lead acetate in the drinking water through gestation and lactation. These data were confirmed by Luthman and colleagues (17), who described delayed eye opening and impairment in negative geotaxis test performance during postnatal ip lead acetate treatment. Grant and colleagues (18) reported significant delays in vaginal opening in female pups and in the development of surface- and free-fall-righting reflexes. Similarly, Livesey and colleagues (19) reported that animals exposed to lead from birth to 20 days of age presented retardation in the development of free-fall righting and acoustic startle reflexes. However, a statistically significant effect of lead treatment on body weight was evidenced in these studies. Since it is well known that undernutrition is causally related to neurobehavioral development retardation (20), it becomes difficult to attribute these deficits solely to lead exposure.

Studies using milder forms of lead intoxication have revealed no differences in weight gain, but significant effects on adult rat behavior $(21,22)$, confirming the view of a deleterious role for lead. However, developmental studies without concomitant undernutrition are still lacking in the literature. Therefore, in the present study we investigated the effects of chronic lead exposure, starting at a prenatal age, on the neurobehavioral development of rats using a protocol of lead intoxication that does not affect weight gain (21).

\section{Material and Methods}

Animals were submitted to prenatal and postnatal lead acetate intoxication as previously described (21). Briefly, female Wistar rats (70-75 days of age, $120-150 \mathrm{~g}$ ) from our breeding stock were given $1.0 \mathrm{mM}$ lead acetate or distilled and deionized water as the sole drinking fluid. Rats were maintained on a natural light cycle in an air-conditioned, constant-temperature colony room. Each cage contained three rats which had free access to lab chow and water or lead acetate solution. After 4 weeks of treatment, rats were placed in breeding groups of three virgin females and one male in an opaque plastic cage $(50 \mathrm{x}$ $25 \times 18 \mathrm{~cm}$ ). Mating lasted 21 days, and pregnant rats were selected and placed in individual cages containing wood shavings as nesting material. Twenty litters were used, eleven of them from females treated with water and nine from females treated with lead acetate. On the day of birth (day 0), the litters were culled to eight pups (4 males and 4 females, whenever possible) and the pups examined for the occurrence of gross malformations. Animals were weighed at 0, 5, 10, 15, 20 and 30 days after delivery.

Reflex tests and observations of the maturation of physical characteristics were carried out daily at the appropriate ages by one experimenter that was not aware of the subject treatment. Testing was carried out between 10:00 and 12:30, and progress of the same individuals from each litter was followed throughout the experiment. The date of appearance of ear unfolding, eruption of upper incisors and eye opening was recorded using previous criteria $(20,23-26)$. The reflexes assessed were righting, palmar grasp, negative geotaxis, cliff avoidance, free-fall righting and auditory startle, as described in detail by Smart and Dobbing (20). Briefly, righting was scored by measuring the time required for the animal to turn ventrally after being placed in a dorsal position from postnatal day 1 to 5; palmar grasp was evaluated 
by stroking gently the palm of a forepaw with a paper clip and observing the digital flexing response. The date of disappearance of the flexing response was recorded. Negative geotaxis was scored as follows: the rat was placed head downwards on a $20^{\circ}$ slope, and scored $0,1,2$, or 3 if it turned to face up the slope $0^{\circ}, 45^{\circ}, 90^{\circ}$, or $135^{\circ}$, respectively, in a 90-s session; cliff avoidance was assessed by placing the animal on the edge of a bench, with nose and forepaws just over the edge. The postnatal day the animal moved away from the edge in three consecutive 30$\mathrm{s}$ trials was recorded. Free-fall righting was recorded as the day the animal turned in midair to land ventrally after being dropped, back downwards, from a $35-\mathrm{cm}$ height onto a cotton wool pad in three consecutive trials, and auditory startle was recorded as the day the animal responded with a sudden and brief extension of the hind limbs to the sound of a mouse trap snapping.

On postnatal day 17 , animals were tested for spontaneous alternation. The apparatus was a T maze (a $35-\mathrm{cm}$ base and two arms each $25 \mathrm{~cm}$ long). Latency to enter one arm of the maze and arm entered were recorded. The animals were tested in consecutive trials (intertrial interval, $20 \mathrm{~s}$ ) until they entered the contralateral arm, with a maximum of 5 trials. When the latency to enter one of the sides was longer than $300 \mathrm{~s}$, the animal was excluded from subsequent testing (23). Percent of failure to alternate was also calculated for each litter and compared between groups. On day 19, the ability to balance and move along the rim of a 2-liter beaker was tested, as described by Smart and Dobbing (20). Latency to escape from the rim and percent of animals that fell from the rim were calculated and compared between groups.

Cerebral, hepatic, and renal lead content were evaluated in some females before mating and in the pups after delivery in order to assess lead intoxication. Kidneys, livers and brains were selected at random, and samples were accurately weighed and placed in a plastic container with $5 \mathrm{ml}$ of nitric acid (27). The mixture was allowed to stand overnight to prevent a too vigorous reaction during microwave heating (28). Thereafter the containers were placed in a domestic microwave oven (Consul, MU31A), where samples were submitted to the following microwave program: 1) four times at $50 \%$ power for $5 \mathrm{~s}$; 2) three times at $50 \%$ power for $10 \mathrm{~s} ; 3$ ) twice at $100 \%$ power for $5 \mathrm{~s}$ (29). After digestion, vessel content was filtered and diluted to 25 $\mathrm{ml}$ (final volume) in a calibrated flask with deionized water.

A Perkin-Elmer 3030-B atomic absorption spectrometer equipped with an HGA400 graphite furnace and an AS-10 autosampler was used to determine lead at the 283.3-nm resonance line (spectral bandwidth $0.7 \mathrm{~nm}$ ) under gas stop and maximum power conditions. A hollow lead cathode lamp operating at $10 \mathrm{~mA}$ was used as the light source. A standard continuous background corrector (deuterium arc bulb) was used. Pyrolytic graphite tubes with L'vov platforms were also used (30). The ashing and atomization temperatures used were 900 and 1800, respectively. A solution of palladium and magnesium was used as a chemical modifier. Measurements were made using an integrated area mode (31).

The data from the date of appearance of each reflex (except for palmar grasp, for which the day of disappearance was recorded) and physical features were analyzed by $2(\mathrm{~Pb}$ or water) $x 2$ (sex) ANOVA using the mean litter score. Negative geotaxis data were analyzed by $2(\mathrm{~Pb}$ or water) x 2 (sex) x 10 (sessions) ANOVA with the session factor treated as within subject factor using the mean litter score. Righting reflex time was analyzed as done for negative geotaxis scores except that the number of sessions was five. Body weight data were analyzed by 2 ( $\mathrm{Pb}$ or water) $\mathrm{x} 2$ (sex) $\mathrm{x} 6$ (measures) ANOVA with the measure factor treated as within subject factor using the mean litter weight. Latency for the first trial in the spontaneous 
alternation test and latency to rim escape were analyzed by $2(\mathrm{~Pb}$ or water) x 2 (sex) ANOVA using the mean litter score. The percent of animals in each litter that failed to alternate in the spontaneous alternation test and that fell from the rim in the beaker test were analyzed by $2(\mathrm{~Pb}$ or water) x 2 (sex) ANOVA. Bartlett's test for homogeneity of variances was run before ANOVA. Cerebral, hepatic and renal lead content data were analyzed by the F-test. $\mathrm{F}$ values are presented only if $\mathrm{P}<0.05$.

\section{Results}

Statistical analysis of data concerning renal, cerebral and hepatic lead content in

Table 1 - Renal, cerebral and hepatic lead content of female rats before mating and of pups after delivery.

${ }^{*} \mathrm{P}<0.05$ compared to respective control ( $F$ values are presented in Results). Results are reported as means $(\mathrm{ppm}) \pm \mathrm{SEM}$, and the number of animals is given in parentheses.

\begin{tabular}{lll}
\hline & \multicolumn{1}{c}{ Control } & \multicolumn{1}{c}{ Lead acetate } \\
\hline $\begin{array}{l}\text { Females } \\
\text { Kidney }\end{array}$ & $0.39 \pm 0.06(5)$ & $1.84 \pm 0.49^{*}(4)$ \\
Liver & $0.88 \pm 0.13(5)$ & $2.43 \pm 0.92^{*}(4)$ \\
Brain & $0.036 \pm 0.006(5)$ & $0.039 \pm 0.004(4)$ \\
Pups & & \\
Kidney & $0.52 \pm 0.30(6)$ & $0.55 \pm 0.16(6)$ \\
Liver & $0.82 \pm 0.58(6)$ & $1.22 \pm 0.12^{*}(6)$ \\
Brain & $0.059 \pm 0.019(6)$ & $0.075 \pm 0.066(6)$
\end{tabular}

Figure 1 - Effect of lead acetate on body weight gain of male and female pups. Data are reported as means \pm SEM of 11 control (water; 42 males and 46 females) and 9 lead acetate-treated litters $(\mathrm{Pb} ; 38$ males and 34 females). ANOVA revealed a significant sex by measure interaction $[F(5,190)=3.31 ; P<0.01]$, indicating that males exhibit a different weight gain curve compared to females.

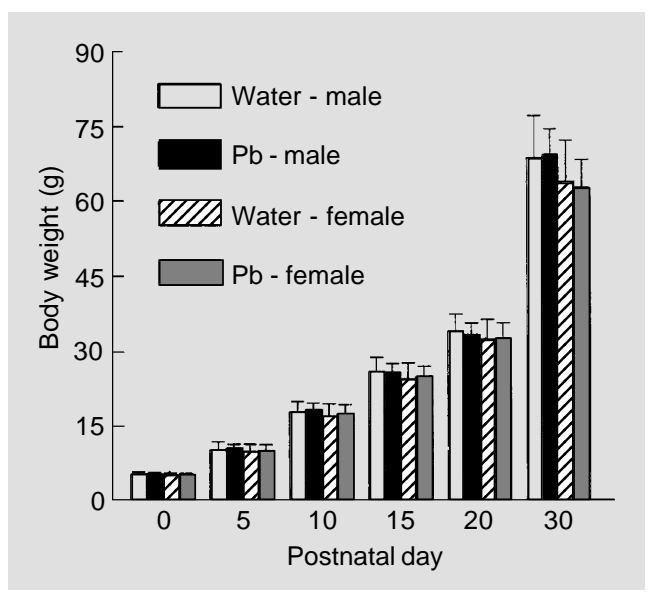

females before mating revealed that animals which had received $1 \mathrm{mM}$ lead acetate in the drinking water presented higher levels of lead in liver $[\mathrm{F}(1,7)=14.2 ; \mathrm{P}<0.005]$ and kidney $[\mathrm{F}(1,7)=48.6 ; \mathrm{P}<0.001]$, but no difference was observed in the brain (Table 1). These results indicate that dams were already intoxicated at mating. Statistical analysis of data about renal, cerebral and hepatic lead content in pups at delivery revealed only an increase of hepatic lead content $[\mathrm{F}(1,10)=5.55 ; \mathrm{P}<0.05]$ (Table 1$)$.

Number of living pups and body weight at birth were similar between groups (data not shown). Lead administration caused no effect on the body weight of the pups. Only a significant sex by measure interaction was observed $[\mathrm{F}(5,190)=3.31 ; \mathrm{P}<0.01]$, suggesting that males exhibit a different weight gain curve compared to females (Figure 1). These results confirm previous studies showing that this protocol of lead intoxication does not affect weight gain $(21,22)$. Statistical analysis of other parameters of physical development revealed that lead treatment caused accelerated eye opening $[\mathrm{F}(1,18)=$ $4.4 ; \mathrm{P}<0.05]$. No difference between groups was observed in the date of ear unfolding or incisor eruption (Table 2).

Statistical analysis of the negative geotaxis data revealed a significant treatment (deionized water or lead acetate) by days interaction $[\mathrm{F}(9,180)=3.11 ; \mathrm{P}<0.005]$, because lead-treated animals presented higher scores and reached a maximal negative geotaxis score before control animals (Figure 2). Statistical analysis also revealed a main effect of treatment for free-fall righting $[\mathrm{F}(1,18)$ $=7.18 ; \mathrm{P}<0.05]$ and auditory startle $[\mathrm{F}(1,18)$ $=7.97 ; \mathrm{P}<0.05]$, indicating that lead administration accelerated the appearance of such reflexes. No significant differences between groups were observed in date of appearance of palmar grasp or cliff avoidance (Table 2) or in time required for righting from a dorsal position during the first five days of life (data not shown). Analysis of beaker rim escape 
latencies revealed no differences between groups in the latency to reach the platform or in the litter percent of falls from the beaker rim (data not shown). Finally, analysis of spontaneous alternation test data revealed a higher percentage of non-alternating animals in the $\mathrm{Ac}_{2} \mathrm{~Pb}$ litters $(37.6 \pm 19.7$ for controls against $57.5 \pm 28.3$ for the $\mathrm{Ac}_{2} \mathrm{~Pb}$ group $\mathrm{F}(1,18)=3.86 ; \mathrm{P}<0.05)$.

\section{Discussion}

In the present study we examined the effect of chronic lead intoxication, initiated before conception, on the development of different behavioral and physical parameters in rats. As previously reported and confirmed in the present study, the addition of $1 \mathrm{mM}$ lead acetate to the drinking water caused no effect on the weight of pups at delivery (21) or on litter size. These results are in agreement with previous reports from our group, which also demonstrated that brain lead levels are similar to those observed in control animals at birth (22). Furthermore, lead acetate administration had no effect on litter weight gain, and therefore undernutrition, a common confounding variable in teratology studies, can be ruled out as a causative agent of the changes observed.

Analysis of other physical development parameters revealed that lead-treated animals presented accelerated eye opening, as also observed in animals subjected to prenatal methylmercury exposure without concomitant undernutrition (32), indicating a teratogenic role for lead acetate. Similarly, the startle reflex appeared earlier in leadtreated animals. In contrast to our study, Luthman and colleagues (17) and Reiter and colleagues (16) reported a delayed eye opening in rats exposed to lead, and Livesey and colleagues (19) found no alteration in this parameter. A common finding reported in these studies was weight gain deficits in lead-treated animals.

Further evidence for a teratogenic role of lead acetate comes from the developmental analysis of different behavioral parameters. Although presenting brain lead levels comparable to the control group at birth, leadtreated animals presented increased scores in the negative geotaxis test. This result also agrees with those reported by Vorhees (32) who studied the effects of prenatal methylmercury intoxication on development without concomitant weight reduction, and are corroborated by the presently observed earlier date of appearance of the free-fall righting reflex. Our results contrast with previous observations by Bull and colleagues (33) and Grant and colleagues (18) who showed that lead-treated animals present a delay in

Table 2 - Postnatal day of appearance of some physical parameters and reflexes.

Palmar grasp is scored as the postnatal day of disappearance of the reflex. Data are reported as mean litter scores ( \pm SEM) of 11 control and 9 $\mathrm{Ac}_{2} \mathrm{~Pb}$ litters. ${ }^{*} \mathrm{P}<0.05$ compared to respective control ( $F$ values are presented in Results).

\begin{tabular}{lcc}
\hline & \multicolumn{2}{c}{ Postnatal day } \\
\cline { 2 - 3 } & Control & Lead acetate \\
\hline Physical parameters & & \\
$\quad$ Ear unfolding & $3.0 \pm 0.7$ & $2.9 \pm 0.2$ \\
Incisor eruption & $9.5 \pm 1.0$ & $9.4 \pm 1.4$ \\
Eye opening & $13.5 \pm 0.6$ & $12.9 \pm 0.6^{*}$ \\
Reflexes & & \\
$\quad$ Cliff avoidance & $4.6 \pm 0.9$ & $4.1 \pm 0.9$ \\
Palmar grasp & $8.1 \pm 1.9$ & $7.6 \pm 1.6$ \\
$\quad$ Startle & $13.0 \pm 0.8$ & $12.0 \pm 0.7^{*}$ \\
Free-fall righting & $14.0 \pm 1.4$ & $12.1 \pm 1.6^{*}$
\end{tabular}

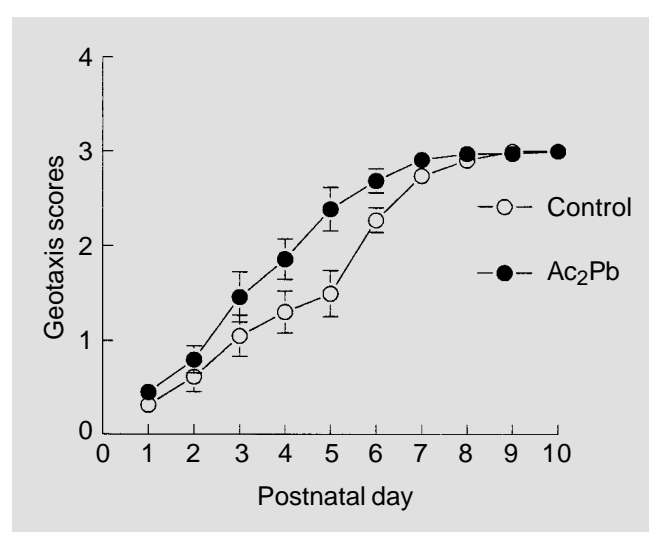

Figure 2 - Effect of lead acetate on the negative geotaxis scores. Data are reported as means \pm SEM of 11 control ( 42 males and 46 females) and $9 \mathrm{Ac}_{2} \mathrm{~Pb}$ litters (38 males and 34 females). $F$ values are presented in Results. 
motor development, including free-fall righting reflex deficits $(18,19)$. However, those studies also reported concomitant body weight gain losses. Similar contradictory results were also observed in animals exposed to methylmercury, since Rocha and colleagues (34) demonstrated that methylmercury-intoxicated animals (which presented weight gain losses) showed impaired performance in the negative geotaxis test, contrasting with data obtained by Vorhees (32). These differences among results may be attributed to different metals, onset, duration and method of imposing heavy metal intoxication, and alterations in maternal behavior, but we think that concomitant undernutrition may be a critical confounding variable in these studies.

It is interesting to point out that two of the behavioral parameters hastened by lead treatment depend on motor responses (negative geotaxis and free-fall righting reflexes). Therefore, it is reasonable to expect that hyperactivity might contribute, at least in part, to the better performance of lead-treated rats in these tasks. However, if hyperactivity were present, one should also expect its influence on other behavioral parameters that depend on motor responses, such as cliff avoidance and beaker equilibration task. These behavioral responses were not affected by lead treatment and do not corroborate the hypothesis that hyperactivity might be involved in this effect. Since spontaneous locomotor activity was not assessed in the present study, the contribution of an increased locomotor response to these results will continue to be speculative.

It is well known that the appearance of the startle reflex coincides with ear channel opening (24-26), and that hearing neuronal circuitry is already developed at this age $(35,36)$. Therefore, earlier ear channel opening is a possible explanation for the earlier occurrence of the startle reflex observed here.

Lead-treated animals present deficits in acquisition of a radial eight arm maze task, performing like hippocampally damaged rats (37). Furthermore, neonatally lead-intoxicated animals present behavioral deficits in the spontaneous alternation test during adulthood (38). In the present study we demonstrated that this deficit occurs at a young age in animals exposed to lead. These results are in agreement with the view that lead treatment causes alterations in hippocampal function (37-41).

In summary, the present study demonstrated that lead acetate intoxication caused significant alterations in the development of rats. The majority of the observed alterations pointed to an advance in the day of appearance of some reflexes, and were in contrast to the deficit observed in the spontaneous alternation test. Similar contradictory advancing and retarding or deleterious effects on rat and hamster development have been demonstrated by others $(26,32)$ using different treatments, and now there seems to be a consensus that any alteration (regardless of direction) in animal development must be considered deleterious $(32,42)$. Therefore, the lead-induced alterations in rat development observed here are important in terms of searching for similar developmental alterations in human beings exposed to low levels of lead, and reinforce the importance of identifying concomitant undernutrition as a confounding variable in experimental teratology studies. Moreover, since subclinical undernutrition may cause biochemical and behavioral alterations in humans, it is highly desirable to evaluate such a condition in animals by measuring other indicators of nutritional status, particularly in studies dealing with chronic administration of neurotoxicants.

\section{Acknowledgments}

We are grateful to Dr. Luciana Malheiros and Dr. João B.T. da Rocha for helpful comments and suggestions. 


\section{References}

1. Bressler JP \& Goldstein GW (1991). Mechanisms of lead neurotoxicity. Biochemical Pharmacology, 41: 479-484.

2. Brown S, Dragann N \& Vogel WH (1971). Effects of lead acetate on learning and memory in rats. Archives of Environmental Health, 22: 370-372.

3. Davis JM, Otto DA, Weil DE \& Grant LD (1990). The comparative developmental neurotoxicity of lead in humans and animals. Neurotoxicology and Teratology, 12: 215-229.

4. Shukla GS \& Singhal RL (1984). The present status of biological effects of toxic metals in the environment: Lead, cadmium and manganese. Canadian Journal of Physiology and Pharmacology, 62: 1015-1031.

5. Cory-Slechta DA (1995). MK-801 subsensitivity following postweaning lead exposure. Neurotoxicology, 16: 83-96.

6. Guilarte TR, Miceli RC \& Jett DA (1995). Biochemical evidence of an interaction of lead at the zinc allosteric sites of the NMDA receptor complex: effects of neuronal development. Neurotoxicology, 16: 63-72.

7. Jett DA \& Guilarte TR (1995). Developmental lead exposure alters $\mathrm{N}$-methyl-Daspartate and muscarinic cholinergic receptors in the rat hippocampus, an autoradiographic study. Neurotoxicology, 16: 7-18.

8. Sandhir R \& Gill KD (1994). Effect of lead on the biological activity of calmodulin in rat brain. Experimental and Molecular Pathology, 61: 69-75.

9. Singh AK (1995). Neurotoxicity in rats chronically exposed to lead ingestion: measurement of intracellular concentrations of free calcium and lead ions in resting or depolarized brain slices. Neurotoxicology, 16: 133-138.

10. Sandhir R, Julka D \& Gill KD (1994). Lipoperoxidative damage on lead exposure in rat brain and its implications to membrane bound enzymes. Pharmacology and Toxicology, 74: 66-71.

11. Davis JM \& Svendsgaard DJ (1987). Lead and child development. Nature, 329: 297300.

12. Annau Z (1990). Behavioral toxicology and risk assessment. Neurotoxicology and Teratology, 12: 547-551.

13. Needleman HL, Schell AS, Bellinger D, Leviton A \& Allred EA (1990). The longterm effects of exposure to low levels of lead in childhood. New England Journal of Medicine, 322: 83-88.
14. McClain RM \& Becker BA (1972). Effects of organolead compounds on rat embryonic and fetal development. Toxicology and Applied Pharmacology, 21: 265-274.

15. Rosenblum WI \& Johnson MG (1968). Neuropathologic changes produced in suckling mice by adding lead to the maternal diet. Archives of Pathology, 85: 640648.

16. Reiter LW, Anderson GE, Laskey JW \& Cahill DF (1975). Developmental and behavioral changes in the rat during chronic exposure to lead. Environmental Health Perspectives, 12: 119-123.

17. Luthman J, Oskarsson A, Olson L \& Hoffer B (1992). Postnatal lead exposure affects motor skills and exploratory behavior in rats. Environmental Research, 58: 236-252.

18. Grant LD, Kimmel CA, West GL, MartinezVargas CM \& Howard JL (1980). Chronic low level lead toxicity in the rat. II. Effects of postnatal physical and behavioral development. Toxicology and Applied Pharmacology, 56: 42-58.

19. Livesey DJ, Livesey RGD, Barrett J \& Spickett TJ (1986). Lead retention in blood and brain after preweaning low-level lead exposure in the rat. Pharmacology, Biochemistry and Behavior, 25: 1089-1094.

20. Smart JL \& Dobbing J (1971). Vulnerability of developing brain. II. Effects of early nutritional deprivation on reflex ontogeny and development of behavior in the rat. Brain Research, 28: 85-95.

21. Rodrigues ALS, Rubin MA, Souza DO \& Mello CF (1993). Lead exposure and latent learning ability of adult female rats. Behavioral and Neural Biology, 60: 274279.

22. Rodrigues ALS, Rocha JBT, Mello CF \& Souza DOG (1996). Effect of perinatal lead exposure on rat behavior in open field and two way avoidance tasks. Pharmacology and Toxicology, 79: 150-156.

23. da-Silva VA, Malheiros LR, Figueiredo LHF, Sá-Rego MM \& Paumgartten FJR (1991). Neurobehavioral development of rats exposed to toluene through maternal milk. Brazilian Journal of Medical and Biological Research, 24: 1239-1243.

24. Da-Silva VA, Smart JL, Freire EM \& Paumgartten FJR (1989). Neurobehavioral development of the golden hamster. Neurotoxicology and Teratology, 11: 105114.

25. Mello CF, Somer JE, Tavaroni V, Graciolli R, Wu V, Torres G, Aguiar F, Wajner M \& Wannmacher CMD (1994). Effects of postnatal methylmalonate administration on neurobehavioral development of rats. Brazilian Journal of Medical and Biological Research, 27: 655-661.

26. Smart JL, Da-Silva VA, Malheiros LR, Paumgartten FJR \& Massey RF (1989). Epidermal growth factor advances some aspects of development but retards others in both rats and hamsters. Journal of Developmental Physiology, 11: 153-158.

27. Subramanian KS (1996). Determination of metals in biofluids and tissues: Sample preparation methods for atomic spectroscopic techniques. Spectrochimica Acta, 51BN: 291-319.

28. Soares ME, Bastos ML \& Ferreira $M$ (1995). Determination of arsenic, cadmium and lead in porcine and bovine kidneys by electrothermal atomic absorption spectrometry. Analyst, 120: 2367-2370.

29. Reid HJ, Greenfield S \& Edmonds TE (1995). Investigation of decomposition products of microwave digestion of food samples. Analyst, 120: 1543-1548.

30. Stoeppler M (1983). Atomic absorption spectrometry - a valuable tool for trace and ultratrace determinations of metals and metalloids in biological materials. Spectrochimica Acta, 51B: 1559-1568.

31. Schlemmer G (1996). Graphite furnace AAS for complex samples: detection limits, precision, long-term stability. Atomic Spectroscopy, 17: 15-21.

32. Vorhees CV (1985). Behavioral effects of prenatal methylmercury in rats: A parallel trial to the collaborative behavioral teratology study. Behavioral Toxicology and Teratology, 7: 717-725.

33. Bull RJ, McCauley PT, Taylor DH \& Crofton KM (1983). The effects of lead on the developing central nervous system of the rat. Neurotoxicology, 4: 1-18.

34. Rocha JBT, Freitas AJ, Marques MB, Pereira ME, Emanuelli $T$ \& Souza DO (1993). Effects of methylmercury exposure during the second stage of rapid postnatal brain growth on negative geotaxis and on delta-amino-levulinate dehydratase in rats. Brazilian Journal of Medical and Biological Research, 26: 10771083.

35. Rodier P (1980). Chronology of neuron development: Animal studies and their clinical implications. Developmental Medicine and Child Neurology, 22: 525-545.

36. Swerdlow NR, Caine SB, Braff DL \& Geyer MA (1992). The neural substrates of sensorimotor gating of the startle reflex: a review of recent findings and their 
implications. Journal of Psychopharmacology, 6: 176-190.

37. Alfano DP \& Petit TL (1981). Behavioral effects of postnatal lead exposure. Possible relationship to hippocampal dysfunction. Behavioral and Neural Biology, 32: 319-333.

38. Kostas J, McFarland DJ \& Drew DG (1976). Lead induced hyperactivity: chronic exposure during the neonatal pe- riod in the rat. Pharmacology, 14: 435 442.

39. Munoz C, Garbe K, Lilienthal H \& Winneke G (1988). Significance of hippocampal dysfunction in low level lead exposure of rats. Neurotoxicology and Teratology, 10: 245-253.

40. Ruppert $P$ (1987). Postnatal development. In: Annau Z (Editor), Neurobehavioral Teratology. Edward Arnold, London.
41. Stoltenburg-Didinger G (1994). Neuropathology of the hippocampus and its susceptibility to neurotoxic insult. Neurotoxicology, 15: 445-450.

42. Tilson HA \& Wright DC (1985). Interpretation of behavioral teratology data. Neurobehavioral Toxicology and Teratology, 7: 667-668. 